1

2

3

4

\title{
Chronic CNS Pathology is Associated with Abnormal Collagen Deposition and Fibrotic- like Changes
}

\section{Daphne Palacios Macapagal, Jennifer Cann, Georgia} Cresswell, Kamelia Zerrouki, Karma Dacosta, Jingya Wang, Jane Connor and Todd S. Davidson

Daphne Macapagal, Bioscience IPF/COPD, Research and Early Development, Respiratory and Immunology (R\&I), BioPharmaceuticals R\&D, AstraZeneca, Gaithersburg, USA Jennifer Cann, Respiratory and Immunology Safety, Clinical Pharmacology \& Safety Sciences, , BioPharmaceuticals R\&D, AstraZeneca, Gaithersburg, USA

Kamelia Zerrouki, Bioscience IPF/COPD, Research and Early Development, Respiratory and Immunology (R\&I), BioPharmaceuticals R\&D, AstraZeneca, Gaithersburg, USA

Karma Dacosta, Clinical Pharmacology \& Safety Sciences, Imaging and Data Analytics, BioPharmaceuticals R\&D, AstraZeneca, Gaithersburg, USA

Jingya Wang, Department of Health and Human Services, Food and Drug Administration, Bethesda, MD USA

Jane Connor, Bioscience IPF/COPD, Research and Early Development, Respiratory and Immunology (R\&I), BioPharmaceuticals R\&D, AstraZeneca, Gaithersburg, USA

Georgia Cresswell, Leica Biosystems, Milton Keynes, UK

Todd S Davidson, Federation Bio, South San Francisco CA 94080 


\section{Abstract:}

27

28 Multiple sclerosis is a chronic debilitating disease of the CNS. The relapsing remitting

29 form of the disease is driven by CNS directed inflammation. However, in the

30 progressive forms of the disease, inflammation has abated and the underlying

31 pathology is less well understood. In this paper, we show that chronic lesions in

32 progressive MS are associated with fibrotic changes, a type of pathology that has

33 previously not thought to occur in the CNS. In an animal model of chronic MS, late

34 stage disease contains no inflammatory infiltrates and is instead characterized by

35 collagen deposition that is histologically similar to fibrosis. In human MS samples,

36 chronic, but not acute lesions, are devoid of inflammatory infiltrates and instead contain

37 significant collagen deposition. Furthermore, we demonstrate that both mouse and

38 human astrocytes are the cellular source of collagen. These results suggest that anti-

39 fibrotic therapy may be beneficial in the treatment of progressive MS. 


\section{Introduction:}

Multiple sclerosis is an autoimmune disease of the central nervous system. Approximately $80-85 \%$ of patients with MS present with a relapsing-remitting (RRMS) course of disease characterized by episodes of disability followed by a period of remission.[1] After a period of years, RRMS can convert into a more progressive disease (secondary progressive, SPMS) in which the patients steadily decline. The remaining $15-20 \%$ of patients with MS will present with a progressive disease from the onset (primary progressive, PPMS)[1,2] RRMS is primarily an inflammatory disease wherein T and B cells, along with macrophages infiltrate the central nervous system (CNS) and cause tissue damage. Such lesions can readily be detected by MRI as gadolinium (Gd) enhancing lesions. Though rare, these lesions are not observed in the progressive forms of disease[3]. In addition, RRMS is amenable to treatment with antiinflammatory treatments, whereas such treatments are generally considered ineffective in treating SPMS and PPMS.

Most MS research has been focused on RRMS as it is the most common form of the disease. Less is known about the progressive forms. One hurdle in studying progressive MS has been the lack of appropriate in vivo models. Experimental autoimmune encephalomyelitis (EAE) has been widely used as an animal model of MS. EAE is induced in susceptible strains of mice by immunization with various myelin derived peptides in adjuvant, resulting in T cell activation, migration into the spinal cord, and subsequent paralysis. This approach has been useful in modeling the immune driven aspects of RRMS, but its highly inflammatory nature would seem to make it inappropriate as a model of progressive MS. 
64 There does not appear to be major genetic differences between RRMS and progressive

65 MS in terms of susceptibility loci, etc. This suggests that the various forms of disease

66 represent stages of the same disease, as opposed to distinct disease entities. We

67 reasoned that EAE may have similar, unappreciated aspects. For example,

68 immunization of B6 mice with MOG35-55 in CFA induces a chronic disease driven by

69 CD4+ T cells. However, the vast amount of research on this model has only focused on

70 the acute phase of disease, while the chronic phase has been relatively ignored.

71 Here, we take an unbiased gene expression approach to characterizing the chronic

72 phase of EAE in the B6 mouse to determine its suitability as a model of SPMS. We

73 determined that chronic EAE is not driven by inflammation, but instead is driven

74 primarily by abnormal remodeling of the extracellular matrix reminiscent of fibrosis. We

75 confirm these findings in human samples and demonstrate their association with various

76 forms of MS. This work demonstrates a previously unappreciated aspect of progressive

77 MS and suggests avenues to future therapies. 


\section{Results:}

Experimental autoimmune encephalomyelitis in the $\mathrm{C} 57 \mathrm{Bl} / 6$ mouse is a well

characterized model of autoimmune mediated neuroinflammation. Often used to demonstrate aspects of multiple sclerosis, it has been studied at the genetic, cellular and molecular level where it has been shown to be driven by autoreactive CD4+ T cells and inflammatory macrophages. Recent advances in genomics technology has enabled characterization of gene expression profiles at unprecedented scale and resolution. Most gene expression studies of EAE have focused on highly purified populations of cells, or even single cell analysis. Moreover, almost all studies have focused on early time points (days 11-23). While analysis of purified cell populations or single cell analysis allows deep understanding of the function of a particular cell type, it necessarily omits changes occurring at the global level.

91 We sought to identify novel pathways regulated during the course of EAE. B6 mice

92 were immunized with MOG35-55 and spinal cords were collected at various time points

93 (Figure 1a). We grouped the disease stage into three phases, pre-disease (days 0 and

94 7), acute disease (days 16 and 23), and chronic disease (days 31 and later). As we

95 were attempting to identify novel pathways associated with disease progression, and

96 not simply changes in gene expression levels due to differences in severity, we carefully

97 selected mice with a clinical score of 3 , characterized by limp tail and paralysis of the

98 hinds limbs, on a scale of 0 to $5(0=$ normal; $5=$ moribund $)$ in the acute and chronic phases of disease. 
100

101

102

103

104

105

106

107

108

109

110

111

112

113

114

115

116

117

118

119

120

121

RNA from the spinal cords of individual mice was analyzed by gene array. The resulting data were filtered to genes with expression levels changed greater than 1.5 fold (normalized to 18s) and with $p$ values less than 0.05 . This resulted in a list of 1477 genes that were significantly regulated. The overlap of genes amongst the various time points can be seen in Figure 1b, with the plurality of genes located in the overlap of days 16,23 , and 31 and thus representing genes that distinguish inflamed from noninflamed CNS. PCA analysis of the significantly regulated genes identified three discreet populations (Figure 1c). Pre-disease spinal cords clustered tightly together, suggesting minimal to no global changes at the gene expression level preceding clinically evident disease. Spinal cords from the acute phase of disease also clustered tightly together, as was expected considering we selected mice with identical clinical scores. Gene expression data suggest that spinal cords from the chronic time point clustered separately from either of the aforementioned populations. This was somewhat unexpected, as we selected mice with identical clinical scores to those in the acute phase of disease. This indicates that although mice in the acute and chronic phase of disease have identical clinical presentations, the underlying gene expression patterns are dramatically different.

This can be visually seen in Figure 1d, where the gene expression patterns between pre-disease and acute disease exhibit an almost inverse relationship. This was likely due to the influx of inflammatory cells that are not normally present in the CNS. The expression pattern at the chronic phase of the disease shows a downregulation of certain genes that were later upregulated in the acute phase. Likewise, an inverse 
122 upregulation of a certain number of genes that were downregulated during the acute

123 phase.

124 We next used Gene Set Enrichment Analysis (GSEA) to identify pathways regulated

125 during different phases of disease. As expected, a number of pathways involved in the

126 inflammatory response were upregulated in acute disease relative to pre-disease.

127 These pathways included the IFN-y response (Figure 1e) and the inflammatory

128 response gene set (Figure 1f). Less well studied in EAE has been IFN-a, but this gene

129 set was also clearly regulated (Figure 1g). However, closer inspection of this particular

130 gene set shows significant overlap with the genes regulated in the IFN- $\vee$ gene set,

131 suggesting that IFN- a may not be the driving factor. Importantly, these three gene sets

132 showed upregulation of key inflammatory genes during the acute disease stage that

133 were subsequently down regulated in the chronic phase.

134 To this point we had identified a clear difference at the gene expression level between

135 acute and chronic disease. The majority of the regulated genes were associated with

136 inflammation and were downregulated at the chronic phase of the disease. Since mice

137 in the acute and chronic phases had identical clinical scores, it was unclear what

138 pathways were being activated to drive pathology at the later stage. We therefore

139 identified GSEA pathways which were upregulated in the chronic phase, but not during

140 the acute or pre-disease stages. We identified only one such GSEA gene set which

141 clearly fit this criteria, epithelial mesenchymal transition (Figure 1h). EMT (Epithelial

142 Mesenchymal Transition) is a pathway in which epithelial cells transform into

143 mesenchymal cells and is one of several mechanisms which can lead to the

144 development of fibrosis. A key driver of EMT is TGF- $\beta$ signaling. We identified 
145 upstream mediators of TGF- $\beta$ signaling in acute disease and downstream mediators of

146 TGF- $\beta$ signaling in chronic disease which clearly fits this criteria (Figure 1i).

147 We first sought to confirm our gene array data and examined more closely the expression profiles of fibrosis associated genes in the CNS. To this end, we designed a

149 Fluidigm panel with an array of fibrosis related probes, including multiple collagen

150 isoforms, genes involved in collagen organization, as well as a number of cytokines

151 known from other organ systems to be involved in inducing collagen synthesis. The

152 panel also included a number of neuronal specific markers and genes involved in the

153 inflammatory response. This analysis included RNA from the original samples used to

154 run the gene array, as well as additional samples isolated from an independent

155 experiment.

156 Hierarchical clustering of the Fluidigm results (Figure 2a) demonstrated clusters of 157 genes regulated at various stages of disease. We confirmed upregulation of

158 inflammatory genes in acute disease relative to pre-disease, followed by downregulation

159 in chronic disease. We also identified a cluster of neuronal markers that were down

160 regulated in acute disease relative to pre-disease. This could be due to death of

161 neurons or could be reflective of the fact that the majority of the RNA in these samples

162 were derived from inflammatory cells thus diluting the neuronal cells RNA in the sample

163 as a whole. Importantly, we saw upregulation of a number of collagen genes, including

164 Col1a1, Col1a2, Col3a1 and Col4a1, all of which are important constituents of fibrotic

165 lesions (Figure 2b). We also saw upregulation of a number of genes involved in

166 organization of the collagen matrix, including Lumican, FAP, FMOD and Serpin1. TGF-

$167 \beta$ and its receptors TGF $\beta R 1$ and TGF $\beta$ R2 were upregulated in both acute and chronic 
168

169

170

171

172

173

174

175

176

177

178

179

180

181

182

183

184

185

186

187

188

189

190

disease. IL-13, also known to be involved in the progression of fibrosis, was upregulated in acute disease along with its receptor IL-13Ra1, while its decoy receptor IL-13Ra2 was downregulated.

EAE is known to be driven by CD4+ Th1 cells and inflammatory macrophages. Our gene expression data suggests that inflammation has abated during the chronic stage of the disease. We confirmed this by flow cytometric analysis of CNS infiltrating leukocytes at various time points (Figure 2c). As stated before, we only selected mice with a clinical score of 3 . We found large numbers of CD45+ cells in the CNS of mice on day 21 , the peak of EAE disease. This number declined by day 28 , and we found almost no CD45+ cells in the CNS on day 42, despite the fact that the mice still had significant clinical disease. Subset analysis of CD45+ cells identified a similar pattern for CD4+IFN- $\mathrm{+} \mathrm{cells,} \mathrm{as} \mathrm{well} \mathrm{as} \mathrm{inflammatory} \mathrm{monocytes,} \mathrm{macrophages,} \mathrm{and} \mathrm{mDCs.}$

Our current understanding of EAE is that it is driven almost exclusively by inflammation. However, these data suggest that during the chronic stage of the disease inflammation has abated and fibrosis drives pathology. This is a common pathway in many diseases, but to our knowledge fibrosis has never been documented in the CNS. We therefore sought to identify fibrotic lesions in the spinal cords of chronic stage EAE mice at the histological level. We isolated spinal cords from mice with EAE at various time points and stained them with Sirius Red (Figure 3a), Masson's Trichrome (Figure 3b) and H\&E (Figure 3c). Sirius red and Masson's trichrome stained sections showed no evidence of fibrosis or abnormal collagen deposition up to day 23. By day 31, abnormal accumulations of collagen were present along the meningeal interface and by day 41 abnormal collagen deposition was clearly present throughout the parenchyma. The 
191 pattern of collagen deposition was clearly abnormal for the CNS, but it was not a classic

192 fibrosis morphology with compact bundles of mature collagen. Perivascular collagen

193 deposition was clearly evident, but the majority of collagen was in the parenchyma and

194 had a fibrillar deposition. H\&E sections demonstrated inflammatory infiltrates in acute

195 disease that had abated by the chronic phase.

196 In most organ systems, fibrosis is mediated by activated fibroblasts. However, the CNS

197 is devoid of such cells, raising the question of which cells produce collagen. We

198 approached this question by co-staining for collagen and various cell types in the CNS

199 of mice with EAE. We found by brightfield microscopy that collagen was associated

200 with astrocytes in diseased, but not normal tissues (Figure 4a). In normal tissue,

201 collagen was associated solely with vessels, and astrocytes were distributed evenly

202 throughout the tissue with characteristic stellate morphology. In mice with chronic EAE,

203 the astrocytes significantly changed their morphology displaying much smaller cell

204 bodies with a fibrillar morphology. To more conclusively demonstrate colocalization of

205 collagen and astrocytes, we analyzed the sections by confocal microscopy (Figure 4b).

206 Non-vascular collagen was found to be significantly, although not exclusively associated

207 with GFAP+ cells.

208 EAE in the B6 mouse is a chronic disease. We have thus far shown that in the later

209 stages of disease, inflammation has abated and the prevailing pathology is fibrotic. We

210 next asked to what extent these fibrotic changes are responsible for the clinical

211 pathology. We and others have previously shown that neutralizing GM-CSF reverses

212 established disease [4] [5] and [6]. Mice treated with anti-GMCSFR have reduced T cell

213 and inflammatory macrophage infiltrates [4]. We treated mice with established EAE 
214 with CAM3003 (anti-GMCSFR) or isotype control and allowed the mice to recover.

215 Spinal cords from mice treated with CAM3003 had fewer Sirius red lesions than mice

216 treated with isotype control (Figure 5a and b). These results suggest that in the chronic

217 stage of disease, GM-CSF signaling is required for the establishment of fibrosis and not

218 the progression of inflammation.

219 To assess collagen content in MS, we immunohistochemically stained sections of brain

220 from human MS patients for collagen-1a (Figure 6a). In all areas of the brain, strong

221 reactivity for collagen-1a was seen in the basement membranes of blood vessels,

222 including small capillaries throughout the neuropil. In chronic demyelinated and sclerotic

223 foci within the white matter, additional fine fibrillar structures and mononuclear cells with

224 fusiform or stellate morphology were also strongly positive for collagen-1a. To

225 determine if these might represent reactive endothelium or attempts at

226 neovascularization, we immunohistochemically stained a separate nearby section for

227 the endothelial cell marker, CD31 (Figure 6b) [7, 8]. In all areas of the brain, moderate

228 to strong reactivity for CD31 was seen in endothelial cells, colocalizing with the

229 underlying collagen-1a+ basement membranes. However, there was no CD31 staining

230 of the fine fibrillar structures or mononuclear cells with fusiform morphology within the

231 sclerotic foci that were strongly positive for collagen-1a. To determine if these might

232 represent astrocytes cell bodies and their processes, we immunohistochemically

233 stained a nearby section with GFAP (Figure 6c). In non-sclerotic areas of the brain,

234 astrocyte cell bodies and their processes were strongly positive for GFAP, but the

235 surrounding cells and neuropil were negative. In the sclerotic foci, strongly positive

236 astrocyte cell bodies and their processes were densely packed and colocalized with the 
bioRxiv preprint doi: https://doi.org/10.1101/2021.07.06.451298; this version posted July 7, 2021. The copyright holder for this preprint (which was not certified by peer review) is the author/funder. All rights reserved. No reuse allowed without permission.

237 collagen-1a+ fine fibrillar structures and mononuclear cells. This suggests that within the 238 glial scar, some astrocytes are actively producing and depositing collagen-1a. 


\section{Discussion:}

240

241

242

243

244

245

246

247 deposition.

248 Fibrosis is the formation of fibrous connective tissue as the result of deposition of

In this study we identified a strong fibrosis associated gene signature in late stage EAE. We demonstrate abnormal collagen deposition at the histological level and show that similar pathological changes happen in human MS samples. These changes only occurred at very late time points in the course of the disease and coincide with a marked reduction in inflammatory infiltrates. Therapeutic intervention with GM-CSFR blocking antibodies ameliorated inflammation and prevented late stage fibrotic changes. Finally, we demonstrated that astrocytes are the source of the abnormal collagen extracellular matrix proteins [9, 10]. Fibrosis has been demonstrated in the meninges [11] [12] yet is thought to not occur in the CNS. Indeed, we found a very distinct pattern of collagen deposition in CNS lesions. These lesions were highly fibrillar and located in chronic resolving lesions. We had considered that the collagen deposition we were seeing was related to neovascularization as such processes are known to take place in a number of other pathological process in the CNS such as traumatic brain injury, stroke and ocular neovascularization [13] [14]. However, we found no co-localization of CD31 with collagen producing cells in MS lesions, suggesting that the collagen deposition observed was not related to neovascularization.

The deposition of collagen could negatively influence reparative processes. For example, oligodendrocyte precursor cells are unable to differentiate into mature myelinating oligodendrocytes on stiff matrices [15]. The role of matrix stiffness has been investigated much more extensively in various fibrotic diseases such as those of 
262 the lung and skin [16-19]. In these instances, stiff collagen matrices dramatically alter

263 the phenotype of resident stromal cells. Our results would suggest that abnormal

264 collagen deposition in the CNS impairs normal physiology.

265 Scarring is a well appreciated feature in MS plaques, but to our knowledge this study is

266 the first demonstration that MS plaques are associated with abnormal collagen

267 deposition. Scarring is associated with astrocytosis and recently astrocytes were shown

268 to adopt a neurotoxic phenotype - termed A1 astrocytes - in MS lesions [20]. Itoh et al

269 have shown in late stage EAE that astrocytes downregulate genes involved in the

270 cholesterol biosynthesis pathway and upregulate proinflammatory genes [21]. In

271 addition, numerous studies have demonstrated dysfunctional astrocytes associated with

272 axonal degeneration [22]. These studies demonstrate that astrocytes play a detrimental

273 role in chronic CNS disease. Our work expands on this concept by showing that

274 astrocytes produce collagen and that this collagen prevents productive repair.

275 In this study we investigated gene expression changes in the spinal cord of mice during

276 different stages of EAE. We carefully selected mice with identical disease scores so

277 that any changes we uncovered were due to the stage of the disease, and not related to

278 severity. We chose to analyze whole spinal cord as opposed to isolated cell

279 populations. Such an approach limits the ability to ascribe any gene changes to a

280 specific cell type but allowed us to identify pathways occurring at a more global scale

281 that may have been missed had we chosen to focus on individual cell populations.

282 GSEA analysis identified pathways that were differentially regulated at various stages of

283 the disease. As expected, acute and peak disease states were associated with a

284 massive upregulation of proinflammatory genes. EAE is well known to be driven by 
285 inflammation, yet at the chronic stage of the disease inflammation has completely

286 abated and despite the fact that the mice continued to display clinical symptoms. The

287 main pathways that were upregulated at these later time points were associated with

288 fibrosis, suggesting that this is the predominant pathway associated with pathology at 289 this stage.

290 Relapsing remitting MS is highly inflammatory and immunomodulatory treatments are 291 relatively successful at controlling disease $[23,24]$. The progressive forms of MS are

292 less inflammatory and have proven to be relatively refractory to immunomodulatory

293 interventions [1, 25]. Progressive MS lesions are characterized by neurodegenerative 294 changes including axonal damage and gliosis [1]. Indeed, it was in these lesions that 295 we identified high levels of abnormal fibrotic changes. Although there are currently no 296 truly effective treatments for progressive MS, a considerable amount of research has 297 been focused on neuroregenerative approaches. Our data suggest that antifibrotic 298 approaches may be beneficial as well. Indeed, it may be that antifibrotic interventions 299 will be required for successful neuroregeneration to occur. 


\section{Figure Legends:}

302 Figure 1: A, Schematic of experimental design. B6 mice were immunized with MOG35-

30355 on day zero and spinal cords were harvested on the indicated days. Mice sacrificed

304 on days 0 and 7 had a clinical score of 0 , while only mice with a clinical score of 3 were

305 selected at the following time points. B, Venn diagram showing the overlap of regulated

306 genes at the indicated time points. C, PCA plot showing clustering of individual mice at

307 the indicated time points for genes regulated at FDR $<0.05$. D. Heatmap display of

308 using the same parameters as in C. E-I, Heatmaps of genes regulated in GSEA genes

309 sets according to disease phase.

310 Figure 2: A, Fluidigm analysis of genes identified in the gene array. Genes were

311 selected based on their appearance in the GSEA gene sets related to collagen,

312 cytokines, and collagen organization. B, Kinetic profiles of select gene expression levels

313 as determined by Fluidigm analysis. Pre-disease indicated in blue, active disease

314 indicated in yellow. C, Total cell counts of the indicated populations in the spinal cord as

315 determined by flow cytometry. Total cells are all CD45+ cells, total CD4 is CD45+CD4+,

$316 \mathrm{CD} 4+\mathrm{IFNg}+$ is CD45+CD4+IFNg+, Tregs are CD45+CD4+Foxp3+, Inflammatory

317 monocytes are CD45+ CD11 ${ }^{+}$CD11c $^{-}$Ly6G $^{-}$Ly6C $^{\text {hi }}$, macrophages are CD45+ CD11 b

318 CD11C Ly6G- $^{-}$

319 Figure 3: B6 mice were immunized for EAE and sacrificed on the indicated days. The

320 clinical score (CS) of each individual animal is indicated. Serial sections were stained

321 with Sirius Red, A, Masson's Trichrome, B, or H\&E, C, to identify areas of fibrosis and to

322 characterize histologically. 
323 Figure 4: Fluorescent microscopy demonstrates co-localization of collagen to GFAP+

324 astrocytes. B6 mice were immunized for EAE and sacrificed at either day 0 (clinical

325 score of 0) or day 54 (clinical score of 3). Spinal cords were collected and processed for

326 fluorescent microscopy by staining with GFAP (yellow), Collagen 1 (red) and DAPI

327 (blue) and analyzed by fluorescent microscopy, A, or by confocal microscopy, B.

328 Figure 5: Anti-GMCSFR prevents the establishment of fibrosis in the CNS. B6 mice

329 were immunized for EAE. At peak of disease (day 14) mice were treated

330 intraperitoneally every other day with $10 \mathrm{mg} / \mathrm{kg}$ anti-GMCSFR (CAM3003) or control until

331 disease subsided. Spinal cords were collected, paraffin embedded and stained with

332 Sirius Red to detect collagen deposition. A, representative images obtained under either

333 brightfield or polarized conditions at 20X magnification. B, each dot represents the

334 average lesions per field for an individual mouse obtained under 10X magnification for

335 mice that received either CAM3003 or control treatments.

336 Figure 6: Collagen deposition in MS plaques. Samples of human MS tissue were

337 stained for collagen-1a, A, CD31, B, or GFAP, C and evaluated by brightfield

338 microscopy. 


\section{Methods:}

341 Induction of Active EAE Disease and Scoring. EAE was induced in 6-9 week old female C57BL/6 mice.

342 On day 0 , animals were immunized subcutaneously in the flanks with $400 \mu \mathrm{g}$ of myelin oligodendrocyte

343 glycoprotein 35-55 (MOG 33-55) (MEVGWYRSPFSRVVHLYRNGK; Anaspec Inc. Catalog No. AS-60130-1)

344 in a 200ul emulsion of Complete Freund's Adjuvant $(4 \mathrm{mg} / \mathrm{ml}$ Mycobacterium tuberculosis; Difco Labs

345 Catalog No. 263810). On day 0 and day 2, 350ng of Pertussis toxin (Bordatella pertussis; Calbiochem

346 Catalog No 516 560-50ug) was injected intra-peritoneally (i.p.). EAE scores were assessed daily for

347 clinical signs of EAE in a blinded fashion. Animals were scored as follows: $0=$ normal; $1=\operatorname{limp}$ tail; $2=$ hind

348 leg paralysis of one leg or difficulty walking/ataxia; 3=paralysis of both hindlimbs (paraparalysis) and tail;

349 4=hindlimbs paraparalysis and one forelimb weakness; 5=moribund (requires sacrifice). All animal

350 procedures were approved by the IACUC board (Institutional Animal Care and Use Committee) of

351 Medimmune Inc. and the protocols followed were in accordance to the guidelines with the Animal

352 Welfare Act (AWA).

353 CNS tissue isolation. Animals were asphyxiated using a lethal dose of $\mathrm{CO}_{2}$. Animals showing no signs of 354 pedal and palpebral reflex were first perfused intracardially using room temperature saline. The spinal 355 cords were isolated and frozen on dry ice. Spinal cords were collected at naïve (score 0, day 0), onset

356 (score 0, day 7), peak (score 3-4, day 16), post peak (score 3, day 23) and chronic (score 3 or higher, day

357 30-onward) clinical points. These samples were stored at $-80^{\circ} \mathrm{C}$ until further processing for RNA

358 extraction. Samples that were to be processed for immunohistochemistry, Sirius red or Masson's

359 Trichrome were placed in $10 \%$ formalin for 24 hours followed by paraffin embedding. Other spinal cords

360 were isolated and frozen at $-80^{\circ} \mathrm{C}$ in Tissue-Tek ${ }^{\oplus}$ O.C.T. compound. These samples will be further

361 processed for IHC.

362 RNA extraction from frozen spinal cords. Each frozen spinal cord from individual mice was processed

363 separately for RNA extraction. Frozen material was placed into a 2mL RNASE/DNASE free Lysing Matrix 
D tube filled with beads (MP Biomedicals Catalog No. 6913-500) for disruption. Instructions were followed using the Qiagen Rneasy ${ }^{\circledR}$ Lipid Tissue Mini Kit (Qiagen Catalog No. 74804) thereafter. Briefly, the spinal cords were homogenized using $1 \mathrm{ml}$ Qiazol ${ }^{\circledR}$ Lysis Reagent (Qiagen Catalog No.79306) and FastPrep-24 5G Homogenizer (MP Biomedicals) for 40 seconds. The lysate was incubated at room temperature for $5 \mathrm{~min}$, topped off with $200 \mu$ l of chloroform (Sigma-Aldrich Catalog No.C2432-500mL), shaken, incubated for $2-3 \mathrm{~min}$ at RT, and centrifuged $\left(12,000 \mathrm{~g}\right.$ at $4^{\circ} \mathrm{C}$ for $\left.15 \mathrm{~min}\right)$. The upper phase was transferred to 1 volume of $70 \%$ ethanol and placed into an RNeasy Mini spin column for centrifugation $(8,000 \mathrm{~g}$ at RT for $15 \mathrm{sec})$. The flow through was discarded and an optional on-column DNAse digestion protocol was followed. Briefly, RNeasy ${ }^{\circledR}$ Mini spin column was washed with $350 \mu$ l of RW1 buffer then centrifuged $(8,000 \mathrm{~g}$ at RT for $15 \mathrm{sec})$. Then $80 \mu \mathrm{l}$ of DNase I incubation mix was added to the spin column $\left(15-25^{\circ} \mathrm{C}\right.$ for $\left.15 \mathrm{~min}\right)$ and a final wash of $350 \mu$ of RW1 buffer followed with centrifugation

$375(8,000 \mathrm{~g}$ at RT for $15 \mathrm{sec})$. RNA was extracted following the final steps of the Qiagen RNeasy ${ }^{\circledR}$ Lipid 376 Tissue Mini Kit as per manufacturer’s instructions. RNA samples were then prepared for Fluidigm ${ }^{\circledR}$ 377 Biomark HD array preparation.

378 Fluidigm Biomark HD array preparation. Total extracted RNA was isolated from each mice spinal cord 379 and used in preparation for Fluidigm ${ }^{\circledR}$ Biomark array. The preparation of RNA was performed with the following steps: (1) cDNA synthesis; (2) cDNA preamp PCR; (3) Biomark HD priming; (4) Biomark assay 381 loading. For cDNA synthesis, $50 \mathrm{ng}$ of total RNA from each sample ( 3 mice per group) was used to 382 initiate the PCR using SuperScript III Reverse Transcriptase (Invitrogen Catalog No. 18080-093). cDNA 383 synthesis was performed as per manufacturer's instructions. Preamplification of cDNA followed using 384 the newly generated cDNA and TaqMan ${ }^{\circledast}$ PreAmp Master Mix Kit (Applied Biosystem Catalog No. 385 4384556). The manufacturer's suggested protocol was followed with minor modifications. $20 \mathrm{X}$ 386 TaqMan ${ }^{\circledR}$ Gene Expression assays (Invitrogen) were combine to a final .2X concentration. These will be used as a pooled assay mix for the PCR. Combined with the TaqMan ${ }^{\circledR}$ PreAmp Master Mix and newly 
generated CDNA, the PCR reaction follows a hold of $95^{\circ} \mathrm{C}$ for $10 \mathrm{~min}$ and a $14 \mathrm{X}$ cycled reaction of $95^{\circ} \mathrm{C}$

for $15 \mathrm{sec}$ and $60^{\circ} \mathrm{C}$ for $4 \mathrm{~min}$. The final solution is dilute 1:5 with DNA Suspension Buffer (10mM Tris,

.1mM EDTA, ph8.0, sterile and DNase/Rnase Tested) (Teknova, Catalog No. T0220).

After samples were preamped, the cDNA will be loaded onto a primed Fluidigm 96.96 Dynamic Array IFC

392 (integrated fluid circuit). Manufacturer's instructions were followed. Briefly, the 96.96 IFC array was

393 primed and loaded onto a HX machine. Samples were then prepared with TaqMan Fast Universal PCR

394 Master Mix (Life Technologies PN435042) and loaded on one end of the array. 20X TaqMan ${ }^{\circledR}$ Gene

395 Expression assays were mixed with 2X Assay Loading Reagent (Fluidigm PN 100-7611) and were loaded

396 on the other end of the array. The 96.96 IFC was loaded onto the HX machine and finally transferred to

397 Biomark HD machine. Results were analyzed using Excel and Qlucore for further analysis.

398 Sirius Red, H\&E and Masson's Trichrome staining. Samples embedded in paraffin were mounted onto

399 glass slides (VWR Micro Slides Catalog no 48311-703) using a rotary microtome (Sakura ${ }^{\circledR}$ Accu-Cut SRM)

400 at a thickness of $5 \mu \mathrm{m}$. After drying slides overnight at $37^{\circ} \mathrm{C}$, slides were stored at RT until further

401 staining with Sirius Red. Prior to using the Picro-Sirius Red Stain Kit (Abcam Catalog No. ab150681),

402 samples were deparaffinized. Briefly, slides were dipped in two different Xylene substitute (Fisherbrand

403 Safe Clear II Catalog No.044-192) consecutively for 5 and $10 \mathrm{~min}$. Then slides were transferred into two

404 washes with $100 \%$ ethanol for 3 min each. This is followed by two washes with $95 \%$ ethanol for 3 min

405 each and a final rehydration step using two washes with deionized water for 5 min each. Following

406 dehydration, slides are stained with Picro-Sirius Red for one hour at RT. Then, the slides are rinsed in

407 acetic acid wash twice for one minute each. Finally, slides are rinsed in 100\% ethanol 3 times for 1 min

408 each and mounted in resin. Slides were also stained with Massons Trichrome using Trichrome Stain

409 (Abcam Catalog No. ab150686). Slides were deparaffinized and hydrated as described previously. The

410 Bouins protocol was not followed. Instead, slides were washed in Weigert's Hematoxylin stain for 5 min

411 at RT followed by a wash of deionized water. Then slides were washed in Biebrich Scarlet/Acid Fuchsin 
412 solution for $15 \mathrm{~min}$ then rinsed in deionized water. Samples were then washed in Phosphomolybdic/

413 Phosphotungstic Acid Solution for 10-15 min and placed in Aniline Blue solution for another 10-15 min.

414 Samples were rinsed in distilled water, 1\% acetic acid solution for 3-5 min, 95\% ethanol twice for $30 \mathrm{sec}$

415 each, and then $100 \%$ ethanol twice for 30 sec each. A final wash using Xylene substitute followed prior

416 to final mounting with synthetic resin.

417 Samples for H\&E staining were first deparaffinized and hydrated. Slides were placed in Hematoxylin for

$4183 \mathrm{~min}$; washed in deionized water twice for 5 min; placed in SelecTech ${ }^{\circledast}$ Define MX-aq (Leica Catalog No.

4193803598 ) for $3 \mathrm{~min}$; washed in water for $1 \mathrm{~min}$; washed in $70 \%$ ethanol for $30 \mathrm{sec}$; stained with eosin for

$42030 \mathrm{sec}$; washed in 100\% ethanol thrice for $1 \mathrm{~min}$ each and placed in Xylene Substitute twice for $3 \mathrm{~min}$

421 each. Slides were then covered slipped.

422 Immunohistochemistry. Frozen sections in OCT were obtained using a Cryostat Thermo Scientific

423 Microm HM550. Sections were mounted onto slides (VWR Micro Slides Superfrost Plus Catalog No

$424 \quad 48311-703)$ at a thickness of $5 \mu \mathrm{m}$. Slides were stored at $-20^{\circ} \mathrm{C}$ until further staining. For anti- mouse

425 collagen staining, individual slides were thawed and dried for $20 \mathrm{~min}$ at RT; fixed using acetone at $-20^{\circ} \mathrm{C}$

426 for 20 min; dried again at RT for 20 min; washed in TBS for 5 min; blocked for two hours at RT with

427 Donkey anti-mouse IgG (Jackson Labs Catalog No.715-007-003); washed 3 times with TBS-T (.05\%

428 Tween) for 2 min each; stained and incubated in a humidified chamber with rabbit anti-mouse Collagen I

429 overnight at $4^{\circ} \mathrm{C}$ (1:100 dilution Abcam Catalog No. ab34710); washed 3 times in TBS-Tween (.05\%

430 Tween) for 5 min each; stained with secondary antibody donkey anti-rabbit AF647 (Abcam Catalog No.

431 150075) for 1 hour at RT; rinsed 3 times with TBS-Tween for 5 min each; and finally mounted using

432 Fluoroshield with DAPI (Sigma Catalog No. F6057-20ml). In conjunction with collagen I staining, anti

433 mouse GFAP staining (astrocyte marker) was used. Anti-mouse GFAP AF594 (1:100 dilution Biolegend

434 Catalog No. 644708).

435 
436 1. M. Bradl and H. Lassmann, Progressive multiple sclerosis. Semin Immunopathol. 31(4), 455-65 (2009).

2. J.R. Wujek, C. Bjartmar, E. Richer, R. M.Ransohoff, M. Yu, V. K. Tuohy, B. D. Trapp, Axon loss in the spinal cord determines permanent neurological disability in an animal model of multiple sclerosis. J Neuropathol Exp Neurol. 61(1), 23-32 (2002).

3. K. Hawker, Progressive multiple sclerosis: characteristics and management. Neurol Clin. 29(2), 423-34 (2011).

4. I. Ifergan, T. Davidson, H. Kebir, D. Xu, D. Macapagal, J. Cann, J. M. Rodgers, Z. N. Hunter, C. L. Pittet, S. Beddow, C. A. Jones, A. Prat, M. A. S, S. D. Miller, Targeting the GM-CSF receptor for the treatment of CNS autoimmunity. J Autoimmun. 84, 1-11 (2017).

5. M. El-Behi, B. Ciric, H. Dai, Y. Yan, M. Cullimore, F. Safavi, G. X. Zhang, B.N. Dittel, A. Rostami, The encephalitogenicity of T(H)17 cells is dependent on IL-1- and IL-23induced production of the cytokine GM-CSF. Nat Immunol. 12(6), 568-75 (2011).

6. J. L. McQualter, R. Darwiche, C. Ewing, M. Onuki, T. W. Kay, J. A.Hamilton, H. H. Reid, C. C. Bernard, Granulocyte macrophage colony-stimulating factor: a new putative therapeutic target in multiple sclerosis. J Exp Med. 194(7), 873-82 (2001).

7. M. J. van Amerongen, G. Molema, J. Plantinga, H. Moorlag, M. J. van Luyn, Neovascularization and vascular markers in a foreign body reaction to subcutaneously implanted degradable biomaterial in mice. Angiogenesis. 5(3), 173-180 (2002).

8. L. V. McIntire, Vascular assembly in engineered and natural tissues. Ann N Y Acad Sci. 961, 246-248 (2002).

9. A. Birbrair, T. Zhang, D. C. Files, S. Mannava, T. Smith, Z. M. Wang, M. L. Messi, A. Mintz, O. Delbono, Type-1 pericytes accumulate after tissue injury and produce collagen in an organ-dependent manner. Stem Cell Res Ther. 5(6), 122 (2014).

10. R. Neary, C. J. Watson J.A. Baugh, Epigenetics and the overhealing wound: the role of DNA methylation in fibrosis. Fibrogenesis Tissue Repair. 8, 18 (2015).

11. J. Sajanti and K. Majamaa, Detection of meningeal fibrosis after subarachnoid haemorrhage by assaying procollagen propeptides in cerebrospinal fluid. J Neurol Neurosurg Psychiatry. 67(2), 185-188. (1999).

12. H. Lassmann, K. Kitz, and H.M. Wisniewski, Histogenesis of demyelinating lesions in the spinal cord of guinea pigs with chronic relapsing experimental allergic encephalomyelitis. J Neurol Sci. 50(1), 109-121 (1981).

13. P. A. Campochiaro, Ocular neovascularization. J Mol Med (Berl). 91(3), 311-221 (2013).

14. S. W. Yu, B. Friedman, Q. Cheng, P. D. Lyden, Stroke-evoked angiogenesis results in a transient population of microvessels. J Cereb Blood Flow Metab. 27(4), 755-763 (2007).

15. T. Lourenço, J. Paes de Faria, C. A. Bippes, J. Maia, J. A. Lopes-da-Silva, J. B. Relvas, M. Grãos , Modulation of oligodendrocyte differentiation and maturation by combined biochemical and mechanical cues. Sci Rep. 6, 21563 (2016).

16. S. Asano, S. Ito, K. Takahashi, K. Furuya, M. Kondo, M. Sokabe, Y. Hasegawa, Matrix stiffness regulates migration of human lung fibroblasts. Physiol Rep. 5(9), 13281 (2017).

17. A. J. Booth, R. Hadley, A. M. Cornett, A. A. Dreffs, S. A. Matthes, J. L. Tsui, K. Weiss, J. C. Horowitz, V. F. Fiore, T. H. Barker, B. B. Moore, F. J. Martinez, L. E. Niklason, E. S. White, Acellular normal and fibrotic human lung matrices as a culture system for in vitro investigation. Am J Respir Crit Care Med. 186(9), 866-876 (2012). 
18. B. Hinz, G. Celetta, J. J. Tomasek, G. Gabbiani,C. Chaponnier, Alpha-smooth muscle actin expression upregulates fibroblast contractile activity. Mol Biol Cell. 12(9), 27302741 (2001).

19. D. Duscher, Z. N. Maan, V. W. Wong, R. C. Rennert, M. Januszyk, M. Rodrigues, M. $\mathrm{Hu}$, A. J. Whitmore, A. J. Whittam, M. T. Longaker, G. C. GurtnerDuscher, Mechanotransduction and fibrosis. J Biomech. 47(9), 1997-2005 (2014).

20. S. A. Liddelow, K. A. Guttenplan, L. E. Clarke, F. C. Bennett, C. J. Bohlen, L. Schirmer, M. L. Bennett, A. E. Münch, W. S. Chung, T. C. Peterson, D. K. Wilton, A. Frouin, B. A. Napier, N. Panicker, M. Kumar, M. S. Buckwalter, D. H. Rowitch, V. L. Dawson, T. M. Dawson, B. Stevens , B. A. Barres, Neurotoxic reactive astrocytes are induced by activated microglia. Nature. 541(7638), 481-487 (2017).

21. N. Itoh, Y. Itoh, A. Tassoni, E. Ren, M. Kaito, A. Ohno, Y. Ao, V. Farkhondeh, H. Johnsonbaugh , J. Burda, M. V. Sofroniew, R. R. Voskuhl, Cell-specific and regionspecific transcriptomics in the multiple sclerosis model: Focus on astrocytes. Proc Natl Acad Sci U S A. 115(2), E302-E309 (2018).

22. M. Cambron, M. D'Haeseleer, G. Laureys, R. Clinckers, J. Debruyne, J. De Keyser, White-matter astrocytes, axonal energy metabolism, and axonal degeneration in multiple sclerosis. J Cereb Blood Flow Metab. 32(3) 413-24 (2012).

23. N.Dargahi, M. Katsara, T. Tselios, M. E. Androutsou, M. de Courten, J. Matsoukas, V. Apostolopoulos, Multiple Sclerosis: Immunopathology and Treatment Update. Brain Sci. 7(7) 78 (2017).

24. O. Torkildsen, K.M. Myhr, and L. Bo, Disease-modifying treatments for multiple sclerosis - a review of approved medications. Eur J Neurol. 23 Suppl 1, 18-27 (2016).

25. A. Abdelhak, M.S. Weber, and H. Tumani, Primary Progressive Multiple Sclerosis: Putting Together the Puzzle. Front Neurol. 8,234 (2017). 
a bioRxiv preprint doi: https:///doi.org/10.1101/2021.07.06.451298; this version posted July 7 2021. The copyright holder for this preprint (which
was not certified by peer review) is the author/funder. All rights reserved. N

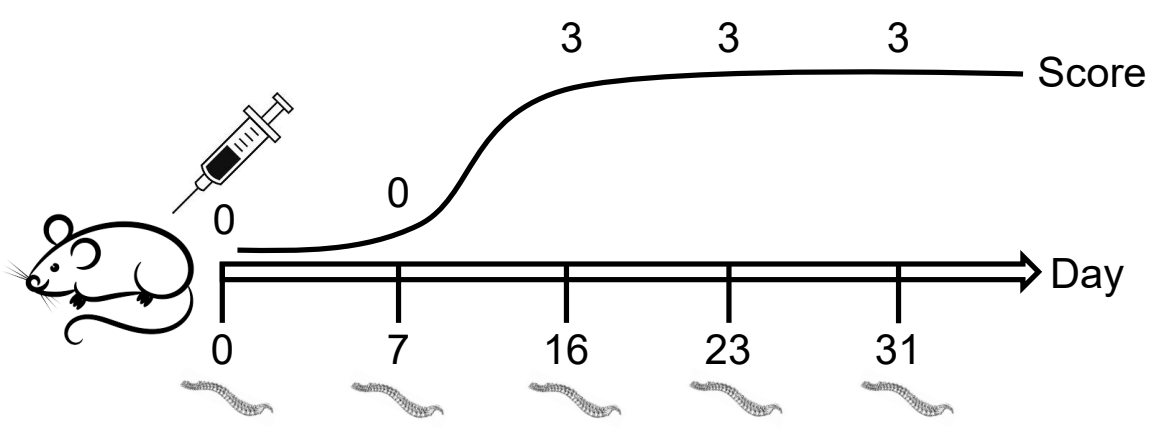

b
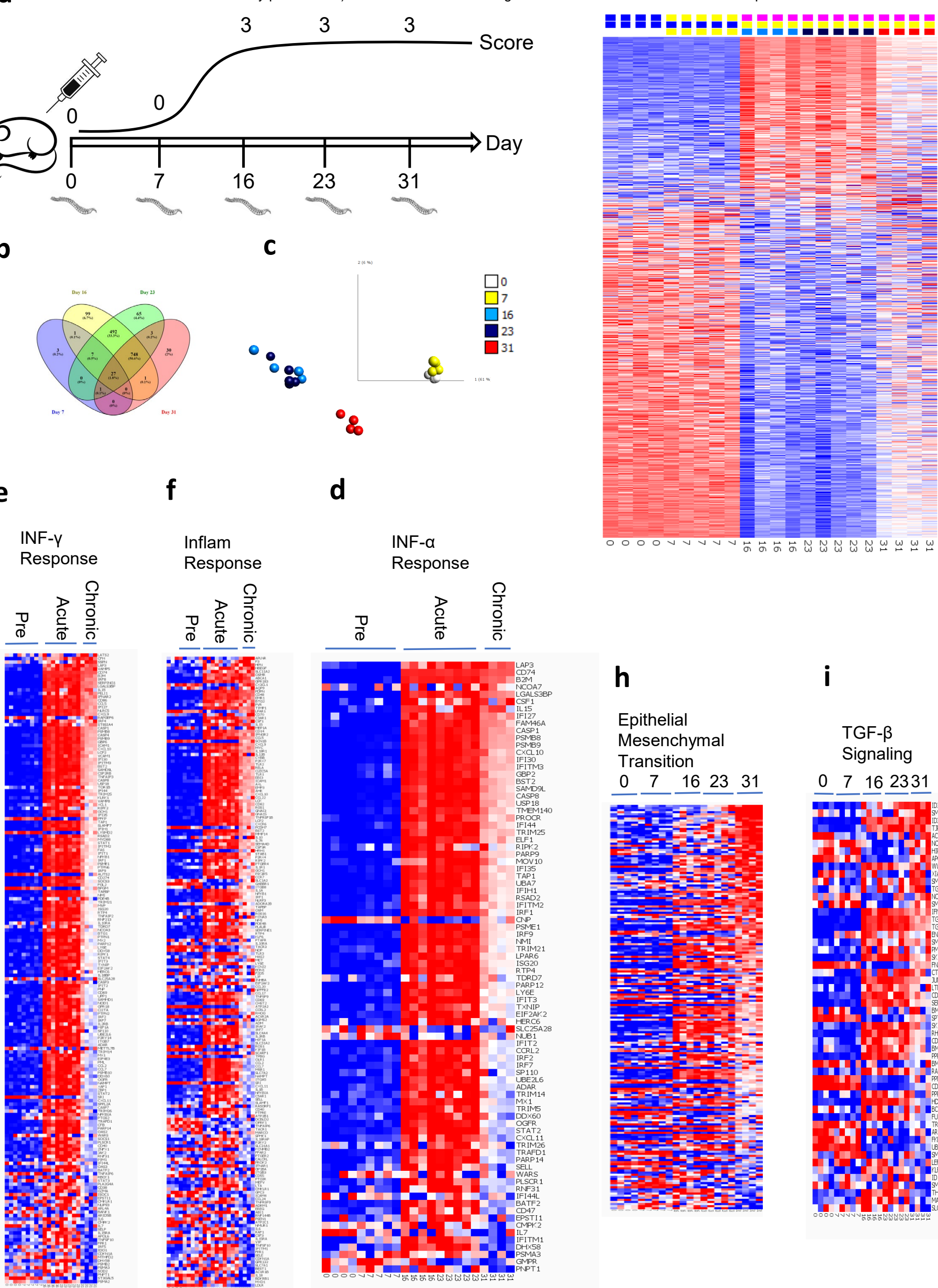

Inflam
Response

INF- $\alpha$

Response

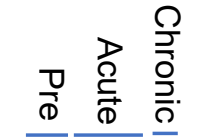
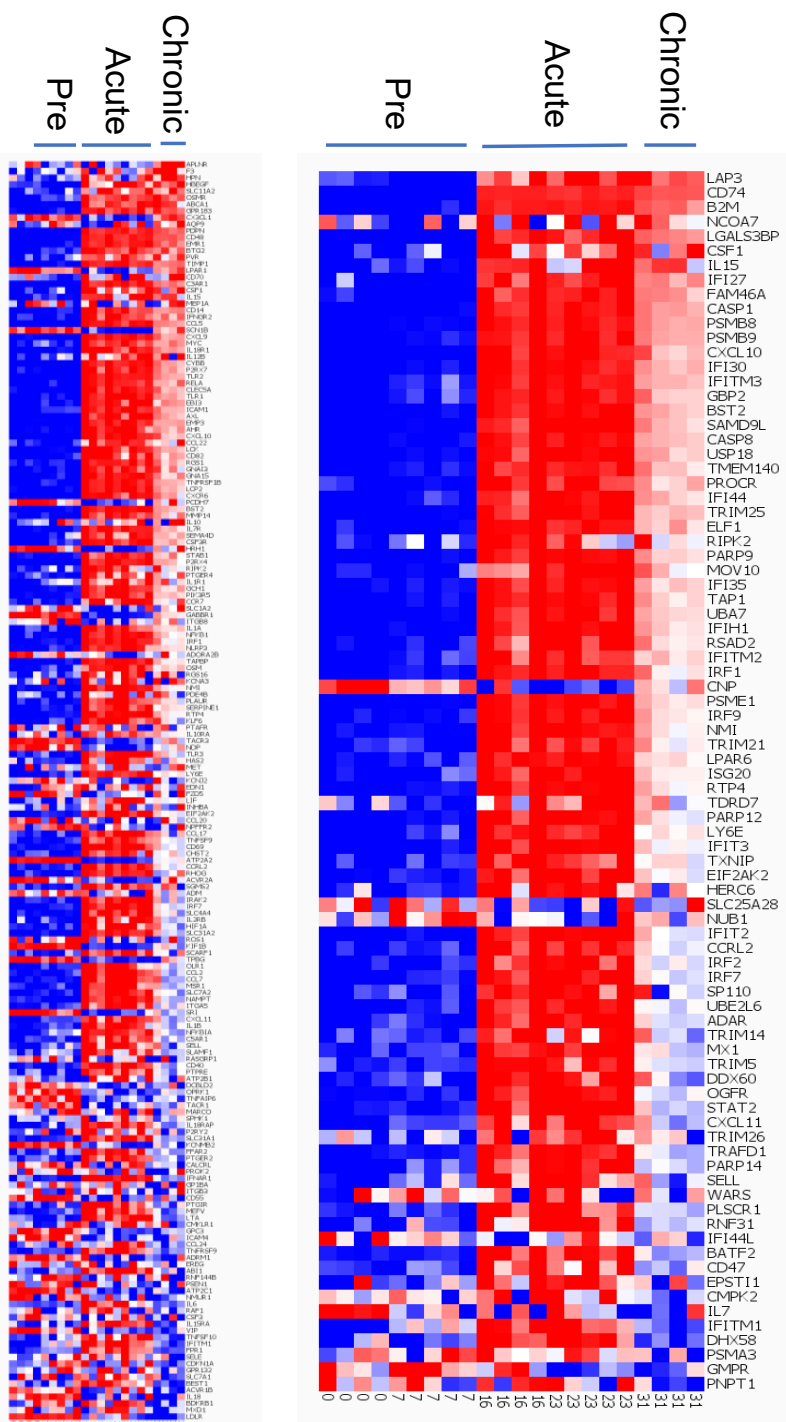

h

Epithelial Mesenchymal Transition

$0 \quad 7 \quad 16 \quad 23 \quad 31$

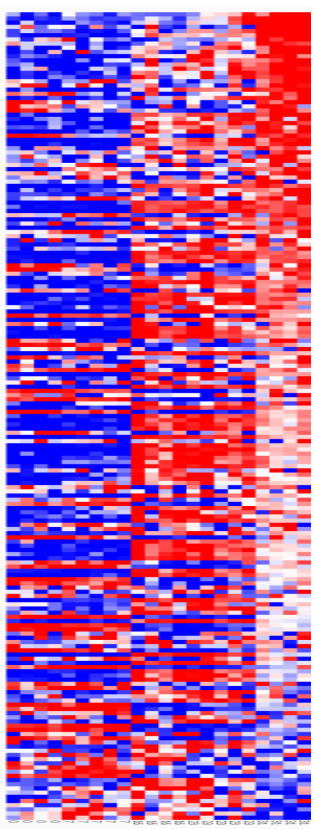

i

TGF- $\beta$

Signaling

$\begin{array}{lll}0 & 7 & 16 \\ 2331\end{array}$

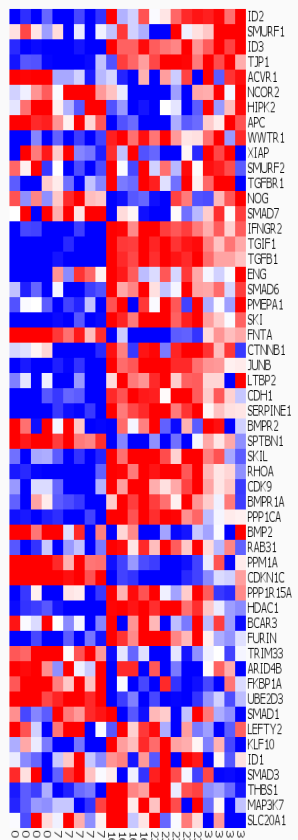


- Figure 1: A, Schematic of experimental design. B6 mice were immunized with MOG35-55 on day zero and spinal cords were harvested on the indicated days. Mice sacrificed on days 0 and 7 had a clinical score of 0 , while only mice with a clinical score of 3 were selected at the following time points. B, Venn diagram showing the overlap of regulated genes at the indicated time points. C, PCA plot showing clustering of individual mice at the indicated time points for genes regulated at FDR $<0.05$. D. Heatmap display of using the same parameters as in C. E-I, Heatmaps of genes regulated in GSEA genes sets according to disease phase. 
a bioRxiv preprint doi: https://doi.org/10.1101/2021.07.06.451298; this version posted July, 2021. The copyright holder for this preprint (which was not certified by peer review) is the author/funder. All rights reserved. Do reuse allowed without permission.

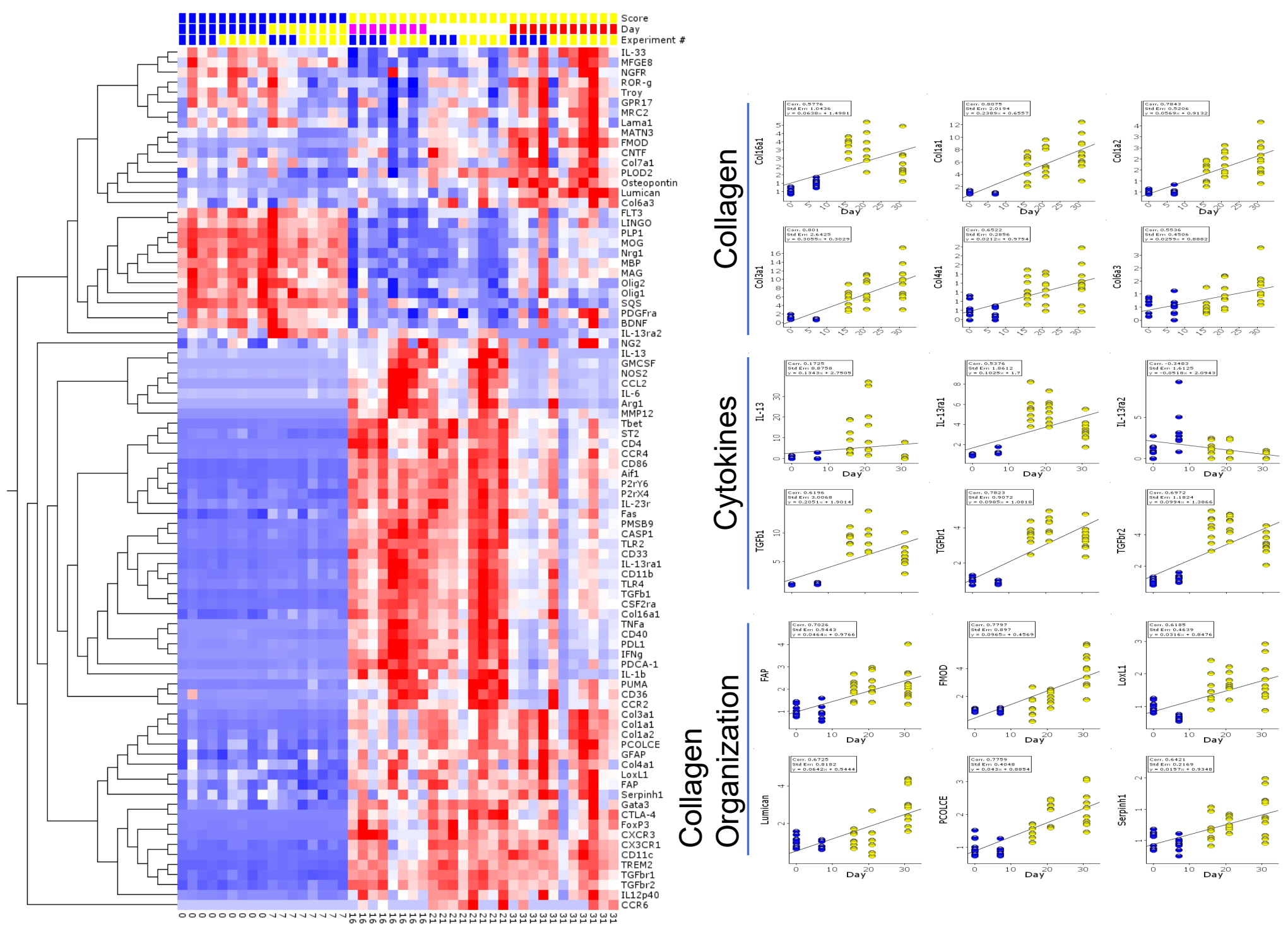

\section{C}
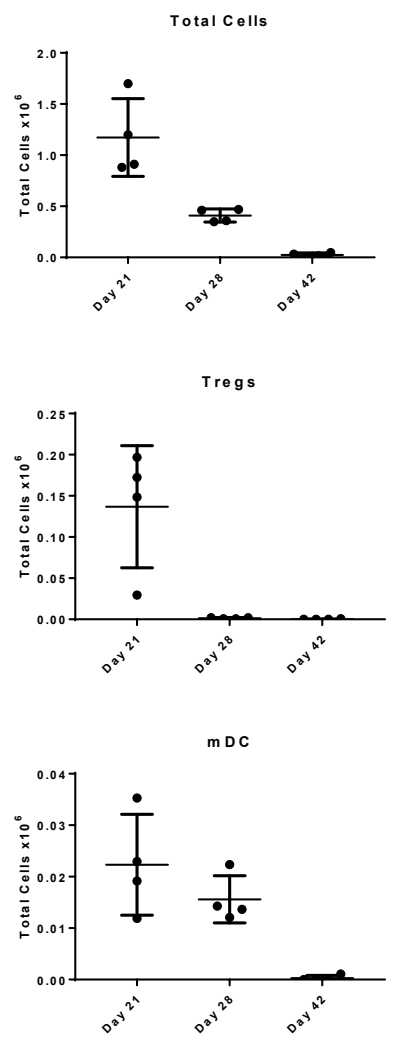
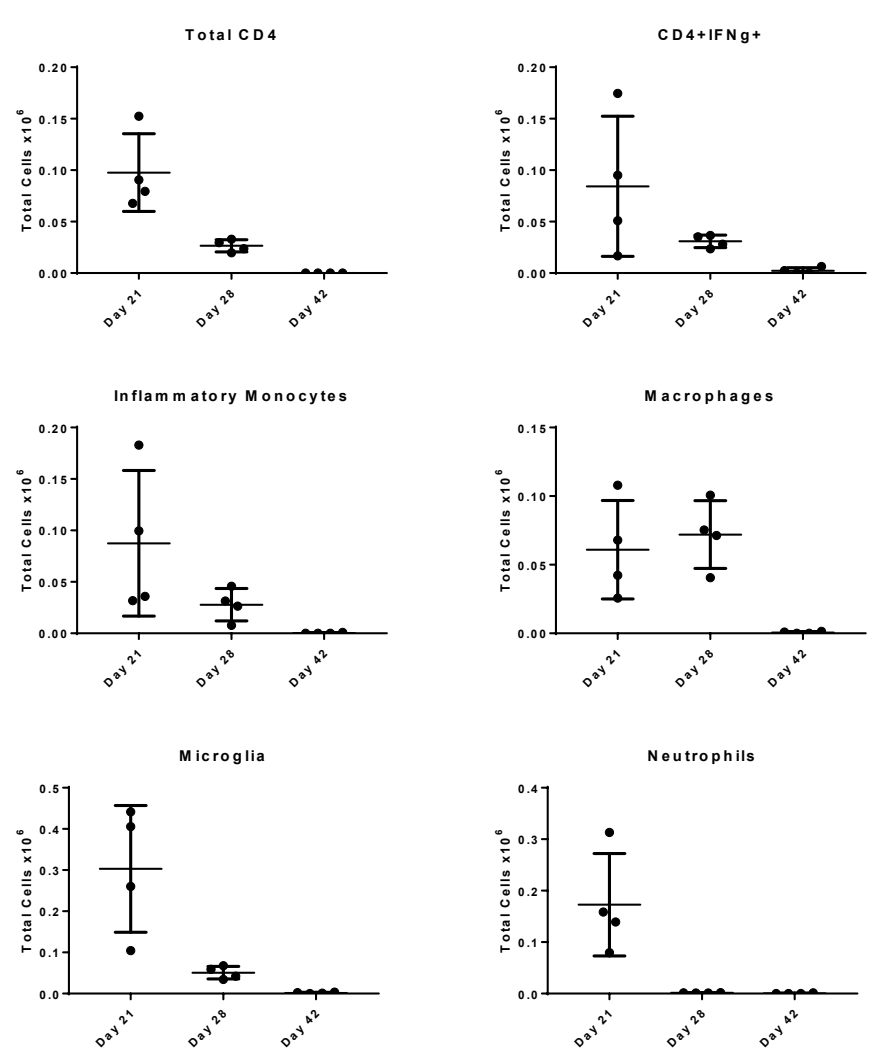
Figure 2: A, Fluidigm analysis of genes identified in the gene array. Genes were selected based on their appearance in the GSEA gene sets related to collagen, cytokines, and collagen organization. B, Kinetic profiles of select gene expression levels as determined by Fluidigm analysis. Pre-disease indicated in blue, active disease indicated in yellow. C, Total cell counts of the indicated populations in the spinal cord as determined by flow cytometry. Total cells are all CD45+ cells, total CD4 is CD45+CD4+, CD4+IFNg+ is CD45+CD4+IFNg+, Tregs are CD45+CD4+Foxp3+, Inflammatory monocytes are CD45+CD11 b+ CD11c- Ly6G- Ly6Chi, macrophages are CD45+ CD11 b+ CD11c- Ly6G- 
bioRxiv preprint doi: https://doi.org/10.1101/2021.07.06.451298; this version posted July 7, 2021. The copyright holder for this preprint (which was not certified by peer review) is the author/funder. All rights reserved. No reuse allowed without permission.

a

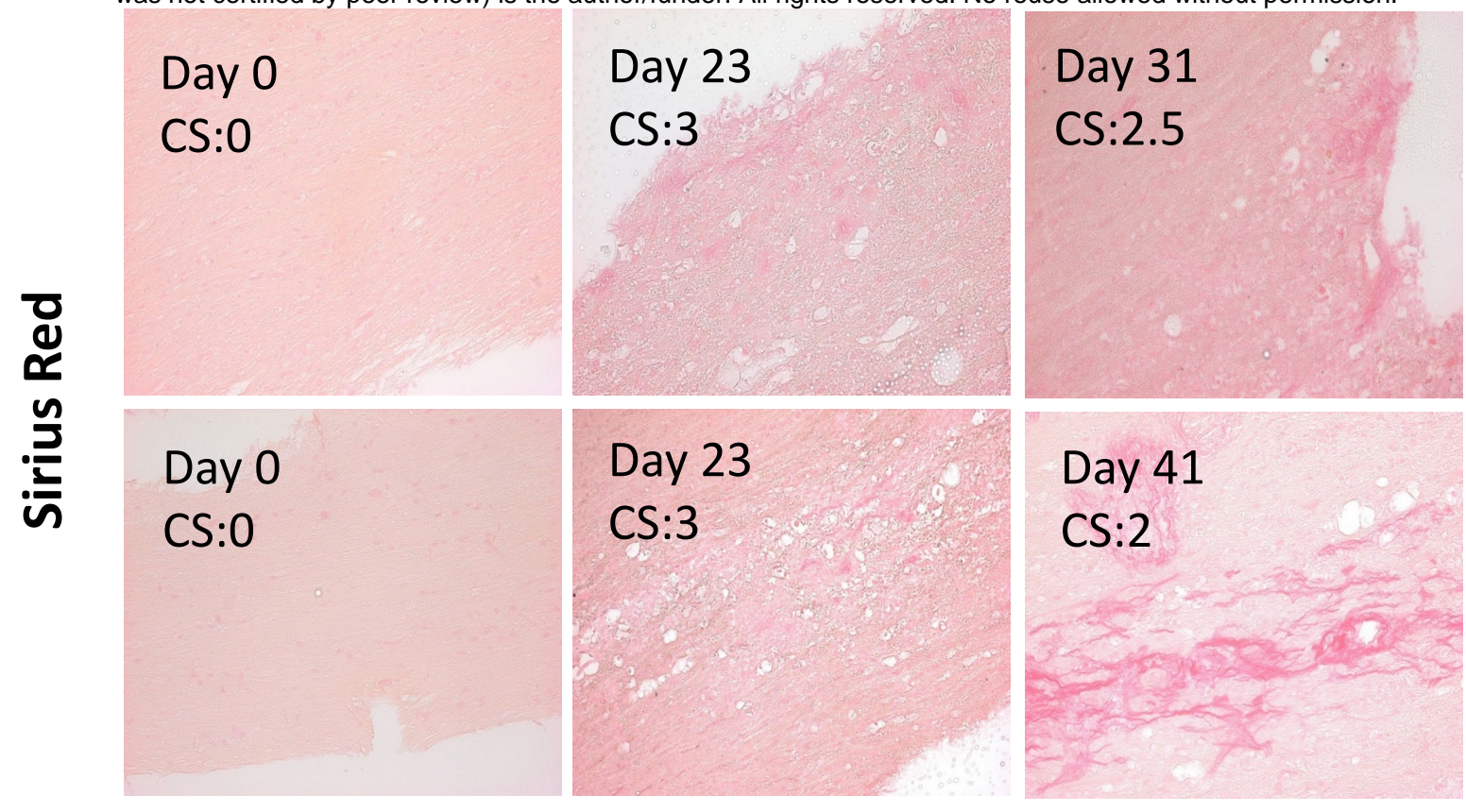

b

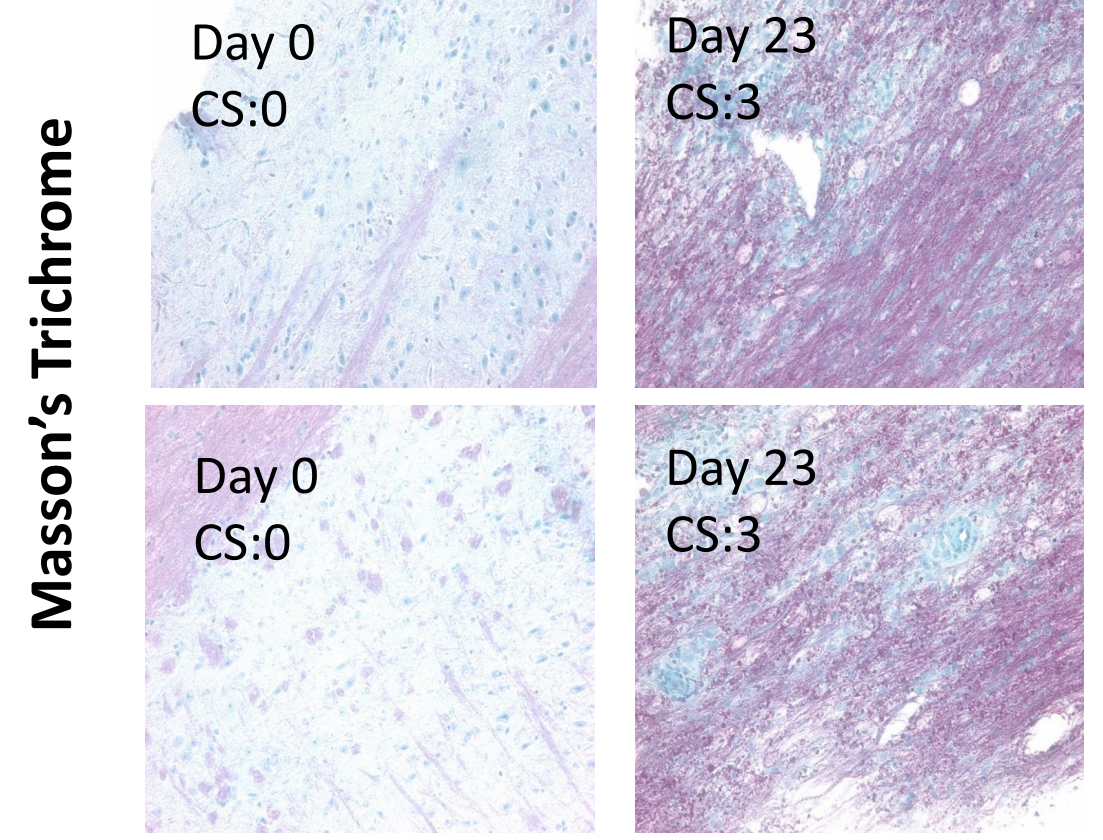

C
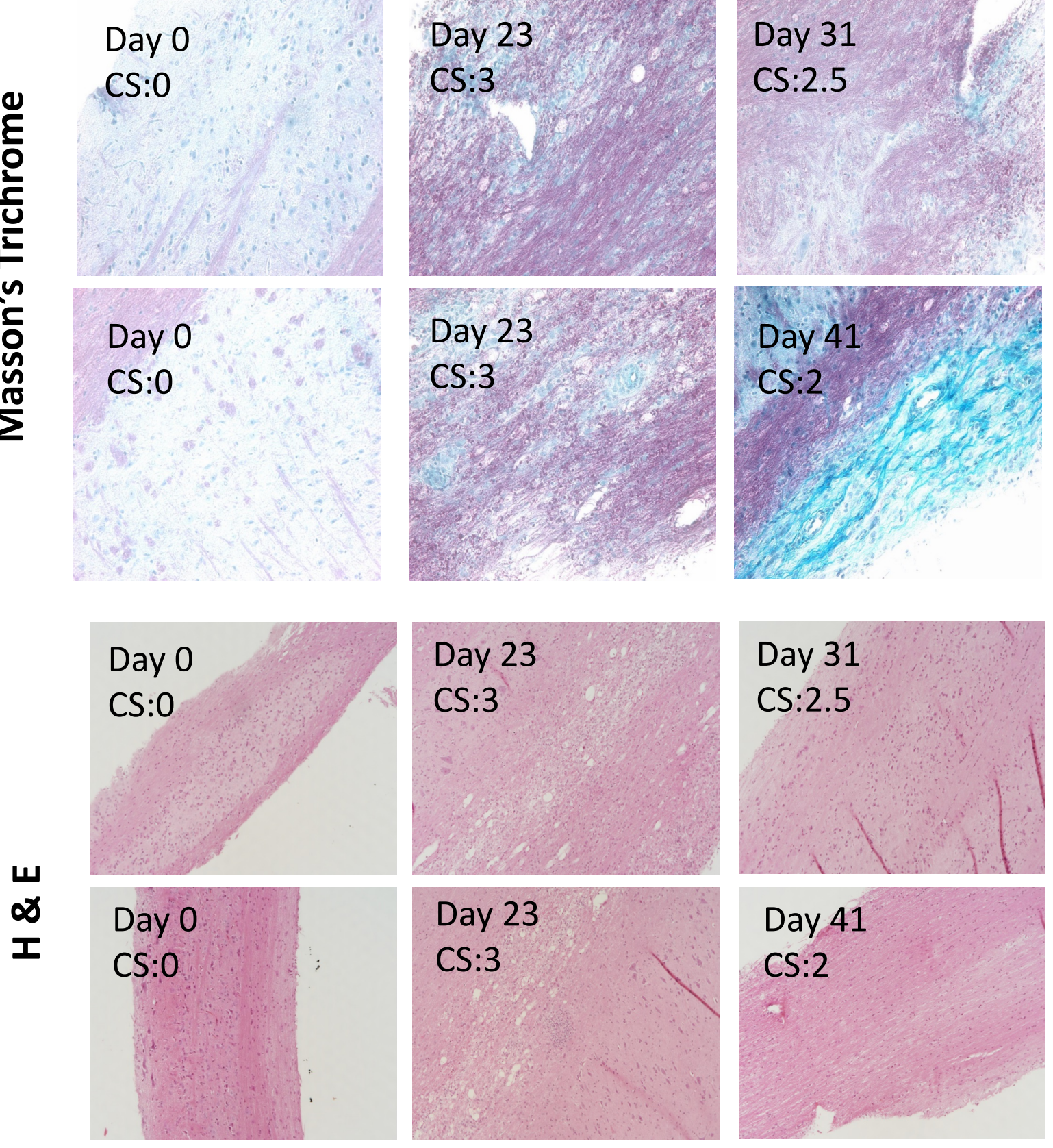

\section{Day 41 \\ CS:2}
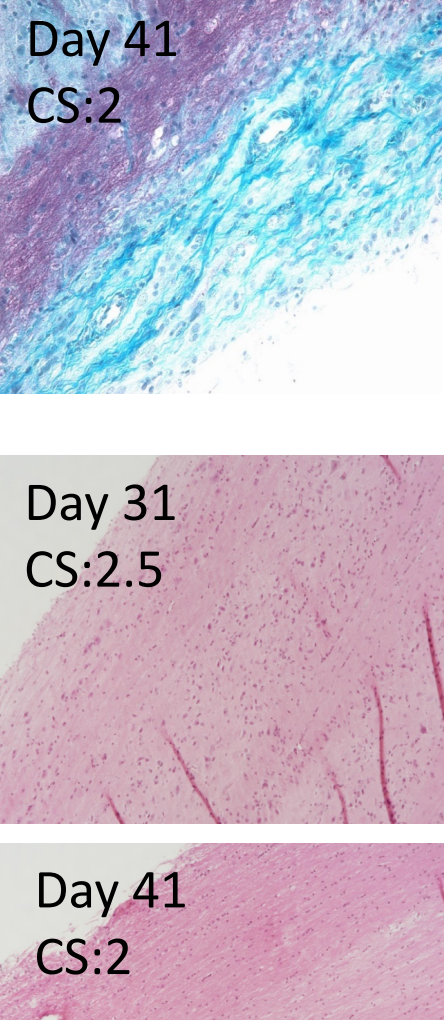
Figure 3: B6 mice were immunized for EAE and sacrificed on the indicated days. The clinical score (CS) of each individual animal is indicated. Serial sections were stained with Sirius Red, A, Masson's Trichrome, B, or H\&E, C, to identify areas of fibrosis and to characterize histologically. 
a

DAPI(blue)
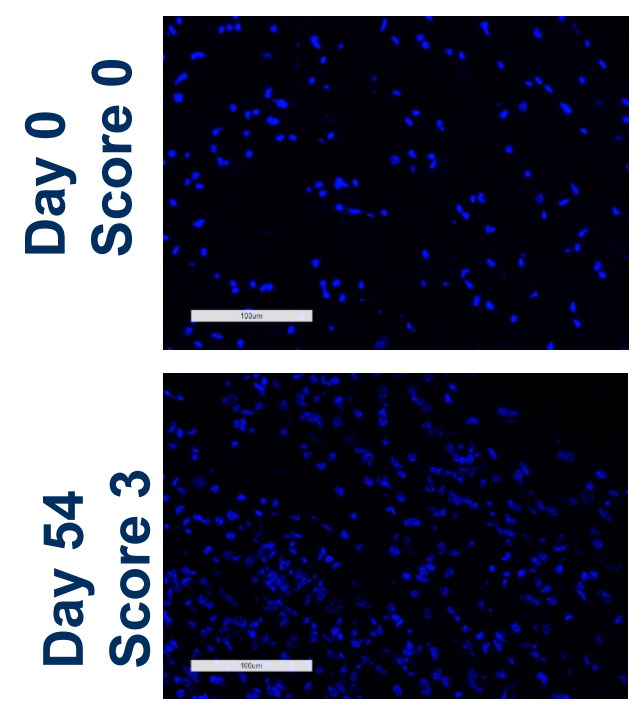

GFAP (Yellow)
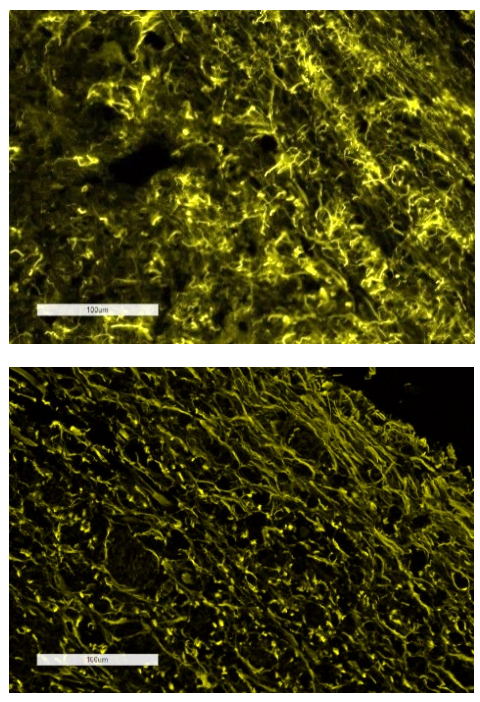

Collagen I (Red)
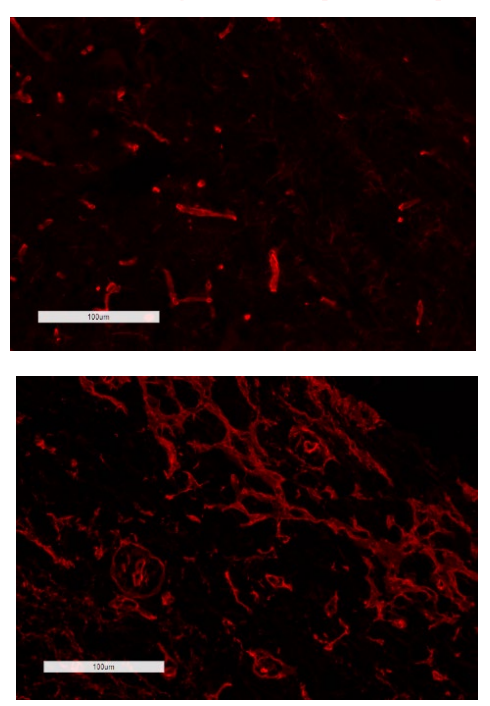

Merge
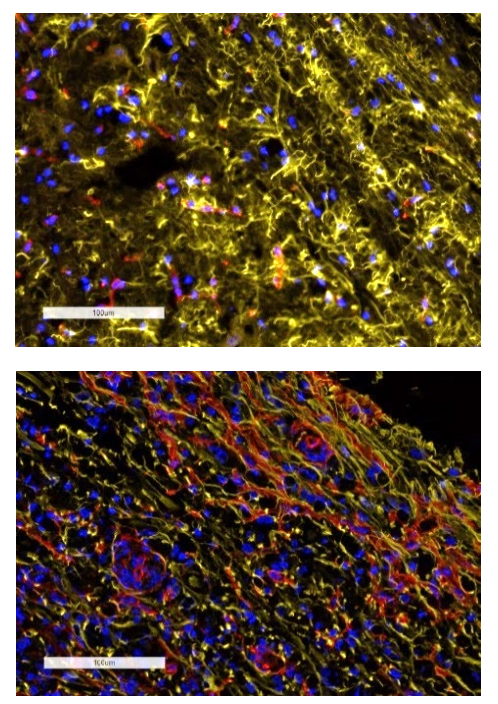

\section{Collagen}

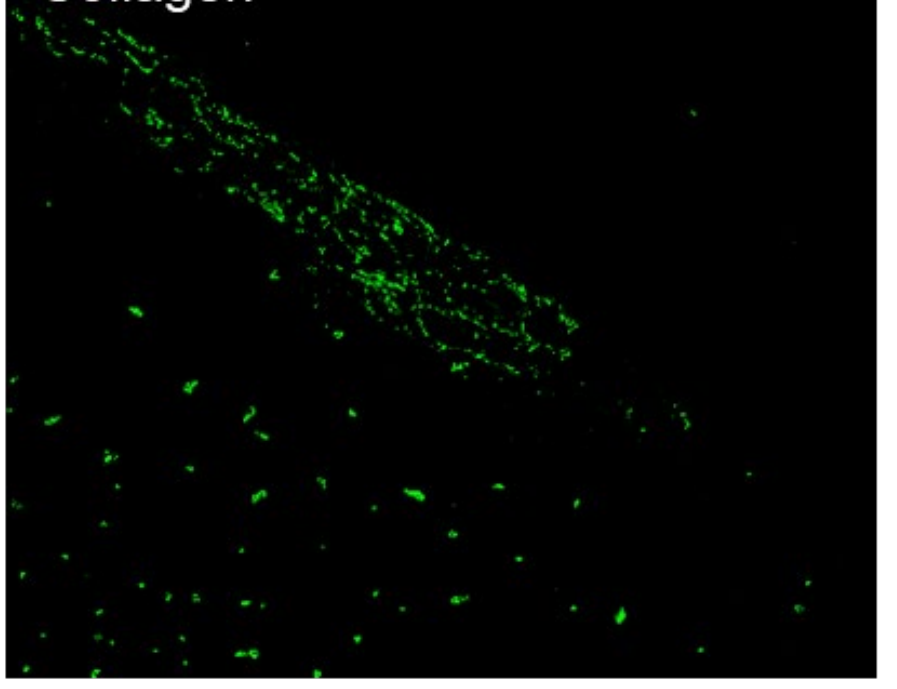

DAPI

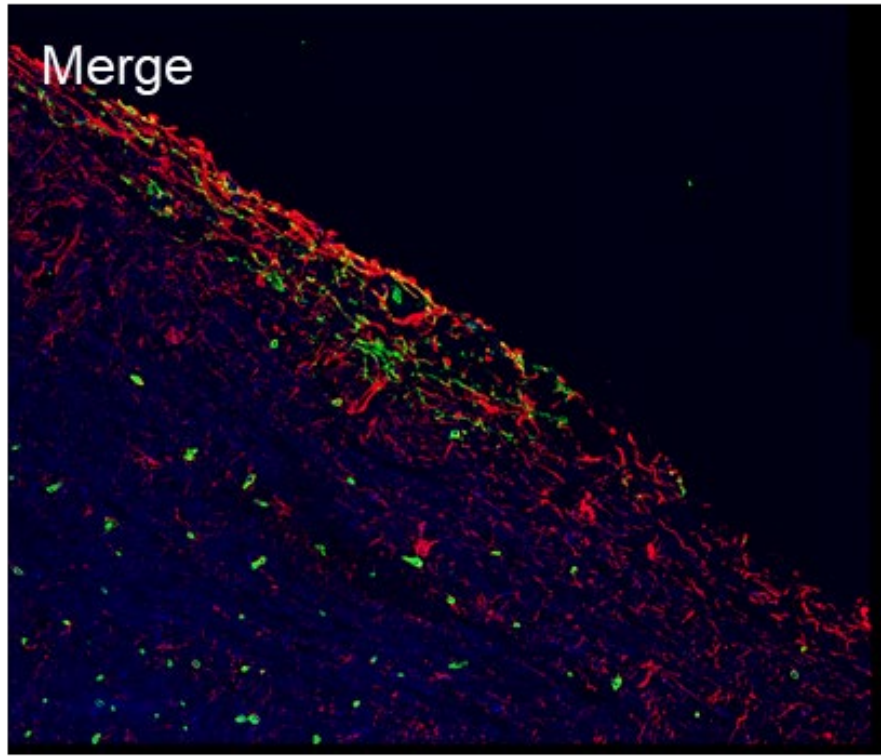

GFAP 


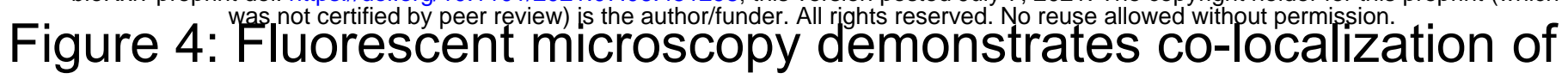
collagen to GFAP+ astrocytes. B6 mice were immunized for EAE and sacrificed at either day 0 (clinical score of 0 ) or day 54 (clinical score of 3). Spinal cords were collected and processed for fluorescent microscopy by staining with GFAP (yellow), Collagen 1 (red) and DAPI (blue) and analyzed by fluorescent microscopy, A, or by confocal microscopy, B. 


\section{Control}
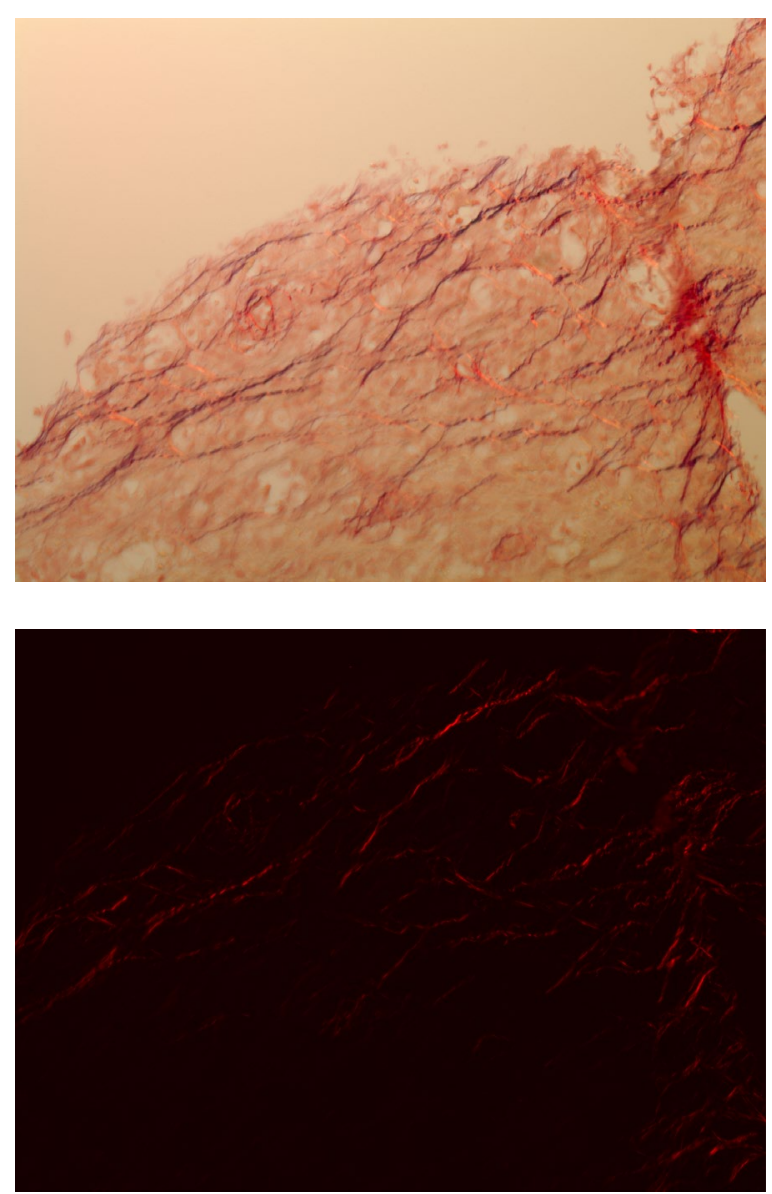

CAM3003 Anti-GMCSFR
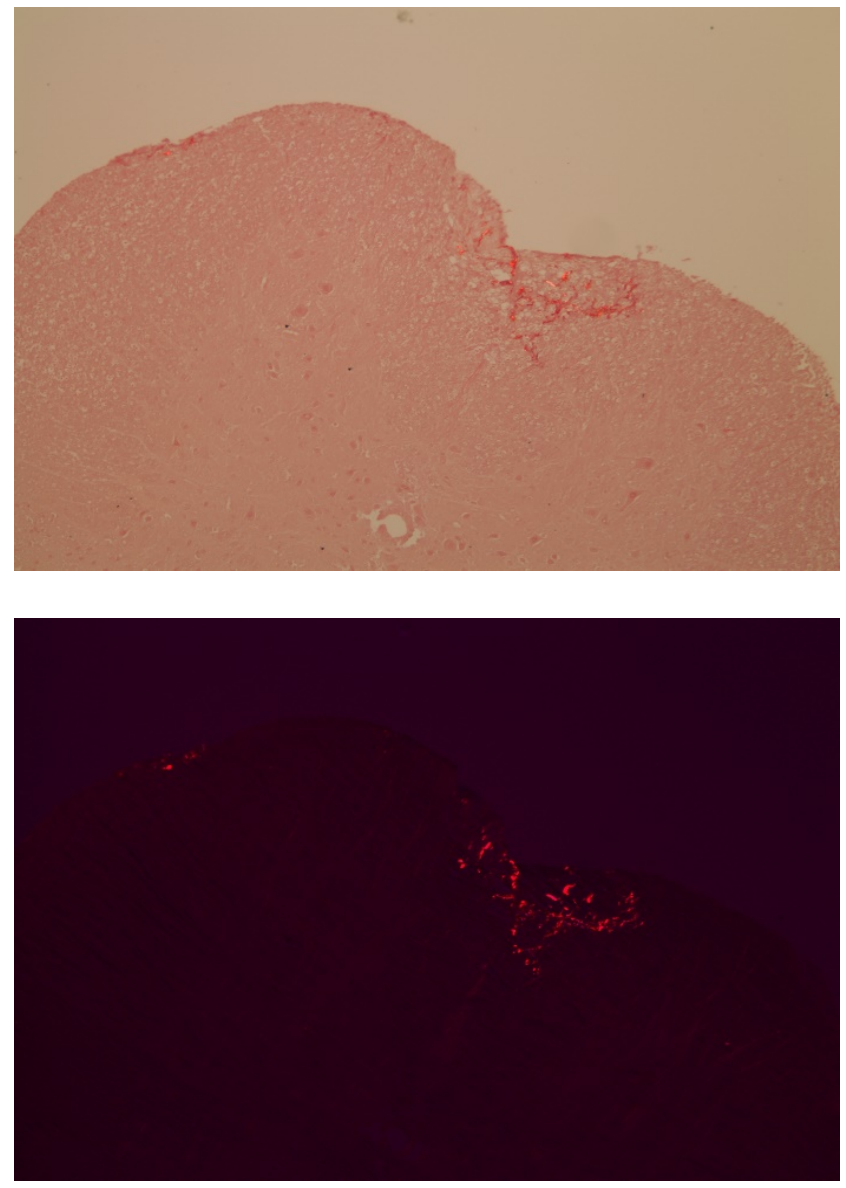

\section{Sirius Red Lesions}

b

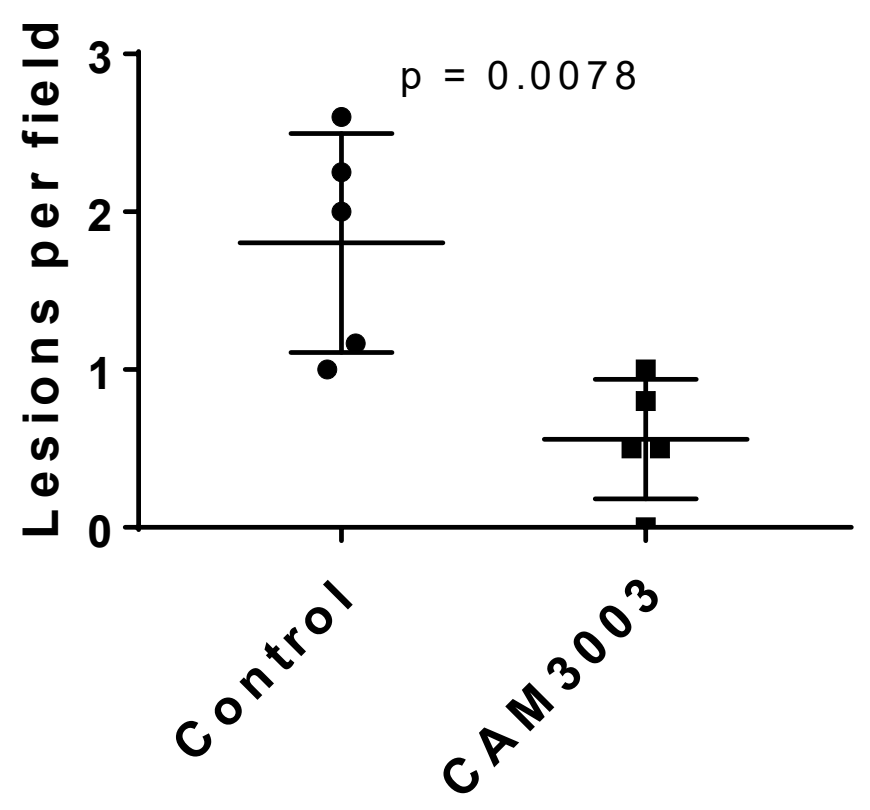


Figure 5: Anti-GMCSFR prevents the establishment of fibrosis in the CNS. B6 mice were immunized for EAE. At peak of disease (day 14) mice were treated intraperitoneally every other day with $10 \mathrm{mg} / \mathrm{kg}$ anti-GMCSFR (CAM3003) or control until disease subsided. Spinal cords were collected, paraffin embedded and stained with Sirius Red to detect collagen deposition. A, representative images obtained under either brightfield or polarized conditions at 20X magnification. B, each dot represents the average lesions per field for an individual mouse obtained under 10X magnification for mice that received either CAM3003 or control treatments. 
bioRxiv preprint doi: https://doi.org/10.1101/2021.07.06.451298; this version posted July 7, 2021. The copyright holder for this preprint (which was not certified by peer review) is the author/funder. All rights reserved. No reuse allowed without permission.

$5 X$ $20 X$

a

\section{Collagen-1a}

b

CD31

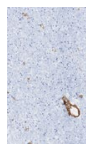

is
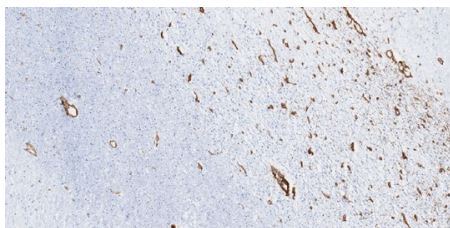

b

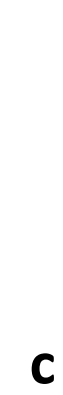

GFAP
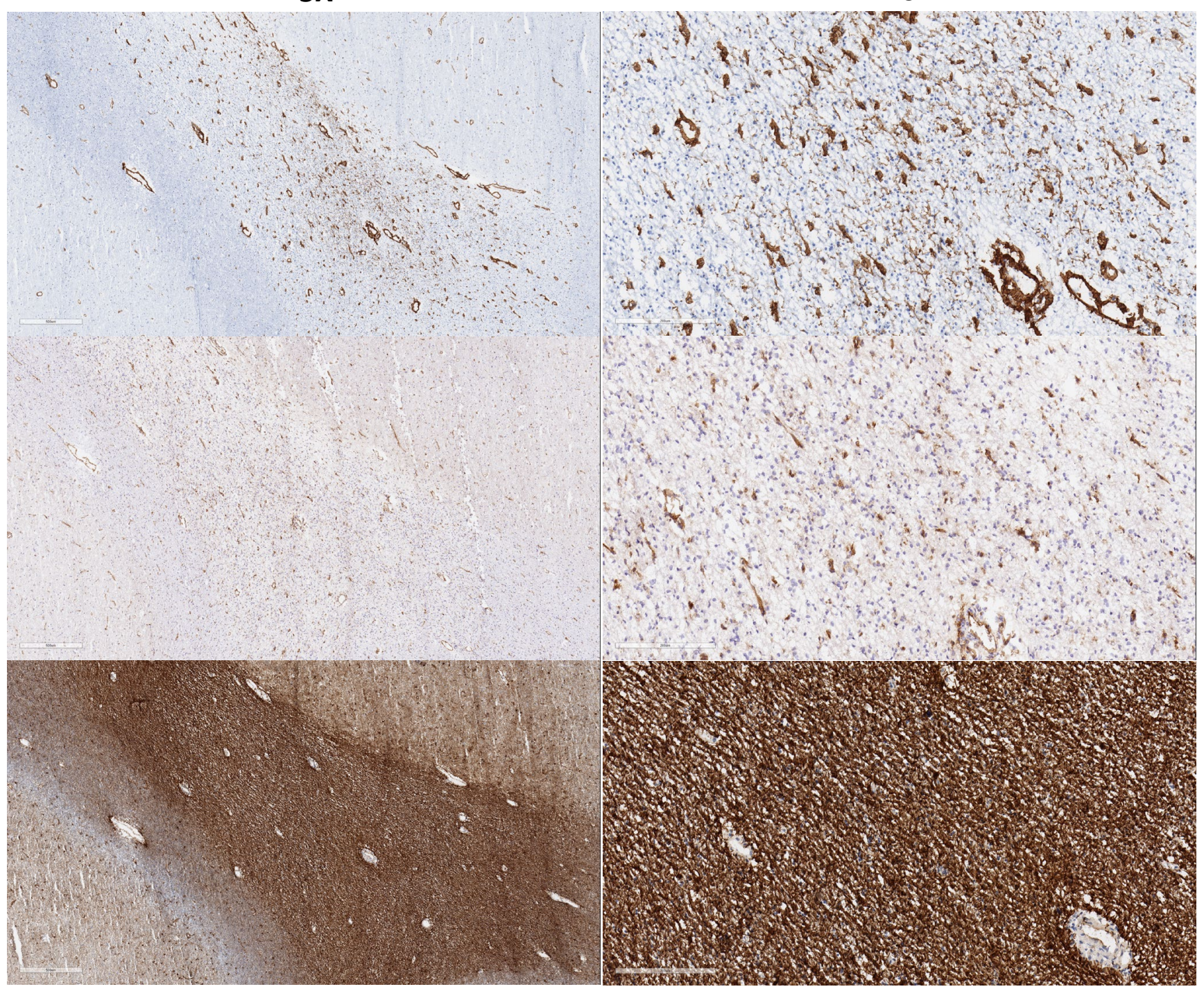

Jennifer Cann, Georgia Creswell

Figure 6: Collagen deposition in MS plaques. Samples of human MS tissue were stained for collagen-1a, A, CD31, B, or GFAP, C and evaluated by brightfield microscopy. 
bioRxiv preprint doi: https://doi.org/10.1101/2021.07.06.451298; this version posted July 7, 2021. The copyright holder for this preprint (which was not certified by peer review) is the author/funder. All rights reserved. No reuse allowed without permission. 
bioRxiv preprint doi: https://doi.org/10.1101/2021.07.06.451298; this version posted July 7, 2021. The copyright holder for this preprint (which was not certified by peer review) is the author/funder. All rights reserved. No reuse allowed without permission. 